\title{
A Note about the Pochhammer Symbol
}

\author{
Aleksandar Petojević
}

ABSTRACT. In this paper we give elementary proofs of the generating functions for the Pochhammer symbol $\left\{(i)_{n}\right\}_{i=0, n \in \mathbb{N}}^{\infty}$.

\section{INTRODUCTION}

For sequence $\left\{c_{n}\right\}_{n=0}^{\infty}$ the generating function, exponential generating function and the Direchlet series generating function, denoted respectively by $g(x), G(x)$ and $D(x)$, are defined as [6, p.3,p.21,p.56]

$$
g(x)=\sum_{n=0}^{\infty} c_{n} x^{n}, \quad G(x)=\sum_{n=0}^{\infty} c_{n} \frac{x^{n}}{n !}, \quad D(x)=\sum_{n=1}^{\infty} \frac{c_{n}}{n^{x}} .
$$

Apart from [6], the relevant theory on generating functions can be found in [1] and Chapter VII in [3].

The Pochhammer symbol $(z)_{n}$ is defined by

$$
(z)_{0}=1, \quad(z)_{n}=z(z+1) \cdots(z+n-1)=\frac{\Gamma(z+n)}{\Gamma(z)},
$$

where $\Gamma(z)$ is the gamma function

$$
\Gamma(z)=\int_{0}^{+\infty} t^{z-1} e^{-t} \mathrm{~d} t \quad(\Re(z)>0) .
$$

For a fixed number $b$ and sequence $\left\{a_{n}\right\}$, the Pochhammer symbol $(b)_{n}$ obeys Euler's transformation

$$
\sum_{n=0}^{\infty} \frac{(b)_{n}}{n !} a_{n} z^{n}=(1-z)^{-b} \sum_{n=0}^{\infty} \frac{(b)_{n}}{n !} \Delta^{n} a_{0}\left(\frac{z}{1-z}\right)^{n},
$$

2000 Mathematics Subject Classification. Primary 11M41, 41A58; Secondary 11B83, $11 \mathrm{~B} 73$.

Key words and phrases. Pochhammer symbol, generating function, Direchlet series, Riemann zeta function, Stirling number, falling factorial.

This work was supported in part by the Ministry of Science and Environmental Protection of the Republic of Serbia under Grant No. 149011D. 
where $\Delta$ is the forward difference defined via $\Delta a_{n}=a_{n+1}-a_{n}$. Higher order differences are obtained by repeated operations of the forward difference operator $\Delta^{k} a_{n}=\Delta^{k-1} a_{n+1}-\Delta^{k-1} a_{n}$, so that in general

$$
\Delta^{k} a_{n}=\sum_{m=0}^{k}(-1)^{m}\left(\begin{array}{c}
k \\
m
\end{array}\right) a_{n+k-m} .
$$

Applying relations (3) and (4) for $a_{n}=1$ to obtain the exponential generating function for the Pochhammer symbol $(b)_{n}$ as follows

$$
\sum_{n=0}^{\infty}(b)_{n} \frac{z^{n}}{n !}=(1-z)^{-b} .
$$

The exponential integral $E_{n}(x)$ is defined by

$$
E_{n}(x)=\int_{1}^{\infty} \frac{e^{-x t}}{t^{n}} \mathrm{~d} t
$$

and has the asymptotic series $[2$, p. 1$]$

$$
(n-1) ! E_{n}(x)=(-x)^{n-1} E_{1}(x)+e^{-x} \sum_{k=0}^{n}-2(n-k-2) !(-x)^{k},
$$

so that

$$
E_{n}(x)=\frac{1}{x e^{x}} \sum_{k=0}^{\infty} \frac{(-1)^{k}(n)_{k}}{x^{k}} .
$$

Hence, generating function is given as follows

$$
\sum_{n=0}^{\infty}(b)_{n} x^{n}=-\frac{E_{b}(-1 / x)}{x e^{1 / x}} .
$$

\section{Statement of Results}

The second possibility of generation of integer sequences by Pochhammer symbol is that for fixed $n \in \mathbb{N}$, terms of the sequence are generated by index $i=0,1,2,3,4, \ldots$, i.e., $\left\{(i)_{n}\right\}_{i=0}^{\infty}$. In this way, here we give for a fixed $n \in \mathbb{N}$ the generating functions for the Pochhammer symbol $\left\{(i)_{n}\right\}_{i=0}^{\infty}$, denoted by

$$
g_{n}(x)=\sum_{i=0}^{\infty}(i)_{n} x^{i}, \quad G_{n}(x)=\sum_{i=0}^{\infty}(i)_{n} \frac{x^{i}}{i !}, \quad D_{n}(x)=\sum_{i=1}^{\infty} \frac{(i)_{n}}{i^{x}} .
$$

First of all, for well-known result

$$
\sum_{i=0}^{\infty}(i)_{n} x^{i}=n ! \frac{x}{(1-x)^{n+1}},
$$

for a fixed number $n \in \mathbb{N}$, we give elementary proof as follows: 
Proof. Let $|x|<1$ and $g_{n}$ be defined by (7). Then

$$
\sum_{i=0}^{\infty}(i)_{n+1} x^{i}=n \sum_{i=0}^{\infty}(i)_{n} x^{i}+\sum_{i=0}^{\infty} i \cdot(i)_{n} x^{i} .
$$

Integrating this equation, we obtain

$$
\begin{aligned}
\sum_{i=0}^{\infty} \frac{(i)_{n+1}}{i} x^{i+1} & =n \sum_{i=0}^{\infty} \frac{(i)_{n}}{i} x^{i+1}+\sum_{i=0}^{\infty}(i)_{n} x^{i+1} \\
\sum_{i=0}^{\infty}(i+1)_{n} x^{i+1} & =n \sum_{i=0}^{\infty}(i+1)_{n-1} x^{i+1}+\sum_{i=0}^{\infty}(i)_{n} x^{i+1} \\
\sum_{i=0}^{\infty}(i)_{n} x^{i} & =n \sum_{i=0}^{\infty}(i)_{n-1} x^{i}+x \sum_{i=0}^{\infty}(i)_{n} x^{i} \\
(1-x) \sum_{i=0}^{\infty}(i)_{n} x^{i} & =n \sum_{i=0}^{\infty}(i)_{n-1} x^{i} .
\end{aligned}
$$

i.e.,

$$
\begin{aligned}
g_{n}(x) & =\frac{n}{(1-x)} g_{n-1}(x)=\frac{n(n-1)}{(1-x)^{2}} g_{n-2}(x) \\
& =\frac{n(n-1)(n-2)}{(1-x)^{3}} g_{n-3}(x)=\cdots=\frac{n !}{(1-x)^{n-1}} g_{1}(x)
\end{aligned}
$$

Now use $g_{1}(x)=x /(1-x)^{2}$, to obtain

$$
g_{n}(x)=n ! \frac{x}{(1-x)^{n+1}},
$$

which completes the proof.

In what follows $\zeta(z), s(n, m)$ and $P_{k}^{n}(x)$ are respectively the Riemann zeta function, Stirling number of the first kind and the polynomials defined by

$$
\begin{aligned}
\zeta(z) & =\sum_{n=1}^{\infty} \frac{1}{n^{z}}, \quad(\Re(z)>1), \\
x(x-1) \cdots(x-n+1) & =\sum_{m=0}^{n} s(n, m) x^{k} \\
P_{k}^{n}(x) & =\sum_{j=0}^{k-1} \prod_{m=0}^{k-j-1}(n-m)\left(\begin{array}{c}
k \\
j
\end{array}\right) x^{j}, \quad(n, k \in \mathbb{N}) .
\end{aligned}
$$

For $\Re(z) \leq 1, z \neq 1$, the function $\zeta(z)$ is defined as the analytic continuations of the foregoing series. Both are analytic over the whole complex plane, except at $z=1$, where they have a simple pole.

We next establish more generating functions given by Theorem 2.1 below. 
Theorem 2.1. For a fixed number $n \in \mathbb{N}$ we have

$$
\begin{aligned}
\sum_{i=0}^{\infty}(i)_{n} \frac{x^{i}}{i !} & =x e^{x}\left[x^{n-1}+P_{n-1}^{n}(x)\right], \\
\sum_{i=1}^{\infty} \frac{(i)_{n}}{i^{x}} & =\sum_{j=1}^{n}(-1)^{j+n} s(n, j) \zeta(x-j) .
\end{aligned}
$$

Proof of (9). Let $G_{n}(x)=x e^{x}\left[x^{n-1}+P_{n-1}^{n}(x)\right]$ and let $[f(x)]^{(k)}$ be the $k^{\text {th }}$ derivative of a function $f(x)$. Since

$$
\begin{aligned}
{\left[G_{n}(x)\right]^{(1)}=x^{n} e^{x}+n x^{n-1} e^{x} } & +e^{x} \sum_{j=0}^{n-2}\left(\begin{array}{c}
n-1 \\
j
\end{array}\right) x^{j+1} \prod_{m=0}^{n-2-j}(n-m)+ \\
& +e^{x} \sum_{j=0}^{n-2}(j+1)\left(\begin{array}{c}
n-1 \\
j
\end{array}\right) x^{j} \prod_{m=0}^{n-2-j}(n-m)
\end{aligned}
$$

induction on $i \in \mathbb{N}$ we have

$$
\begin{aligned}
{\left[G_{n}(x)\right]^{(i)}=e^{x} x^{n} } & +e^{x} \sum_{j=1}^{i}\left(\begin{array}{c}
i \\
i-j
\end{array}\right) x^{n-i} \prod_{m=0}^{j-1}(n-m)+ \\
& +e^{x} \sum_{j=0}^{n-2}\left(\begin{array}{c}
n-1 \\
j
\end{array}\right) x^{j+1} \prod_{m=0}^{n-2-j}(n-m)+ \\
& +e^{x} \sum_{s=0}^{i-1}\left(\begin{array}{c}
i \\
s+1
\end{array}\right) \sum_{j=s}^{n-2} \frac{(j+1) !}{(j-s) !}\left(\begin{array}{c}
n-1 \\
j
\end{array}\right) x^{j-s} \prod_{m=0}^{n-2-j}(n-m) .
\end{aligned}
$$

Hence

$$
\begin{aligned}
{\left[G_{n}(0)\right]^{(i)} } & =\sum_{s=0}^{i-1}\left(\begin{array}{c}
i \\
s+1
\end{array}\right)(s+1) !\left(\begin{array}{c}
n-1 \\
s
\end{array}\right) \prod_{m=0}^{n-2-s}(n-m) \\
& =i !(n-1) ! n ! \sum_{s=0}^{i-1} \frac{1}{(i-s-1) !(s+1) !(n-s-1) ! s !} \\
& =i !(n-1) ! n ! \cdot \frac{(n+i-1) !}{i !(i-1) ! n !(n-1) !}=\frac{(n+i-1) !}{(i-1) !}=(i)_{n} .
\end{aligned}
$$

Applying the standard formula for the Taylor series expansion about the point $x=0$ we arrive at the formula in (9), which completes the proof.

Proof of (10). Using

$$
\sum_{i=1}^{\infty} \frac{(i)_{n+1}}{i^{x}}=n \sum_{i=1}^{\infty} \frac{(i)_{n}}{i^{x}}+\sum_{i=1}^{\infty} \frac{(i)_{n}}{i^{x-1}}
$$


we have

$$
D_{n+1}(x)=n D_{n}(x)+D_{n}(x-1) .
$$

The recurrence relation for Stirling numbers of the first kind

$$
s(n+1, j)=s(n, j-1)-n s(n, j)
$$

produces

$$
\begin{aligned}
& \sum_{j=1}^{n+1}(-1)^{j+n+1} s(n+1, j) \zeta(x-j)= \\
& \quad=n \sum_{j=1}^{n}(-1)^{j+n} s(n, j) \zeta(x-j)+\sum_{j=1}^{n}(-1)^{j+n} s(n, j) \zeta(x-1-j) .
\end{aligned}
$$

Induction on $n$ and by combining (11) and (12) we obtain the result of the theorem.

Note 1. For $1 \leq k \leq 4$ the polynomials $P_{k}^{n}(x)$ are listed below.

$P_{1}^{n}(x)=n$

$P_{2}^{n}(x)=2 n x+n(n-1)$

$P_{3}^{n}(x)=3 n x^{2}+3 n(n-1) x+n(n-1)(n-2)$

$P_{4}^{n}(x)=4 n x^{3}+6 n(n-1) x^{2}+4 n(n-1)(n-2) x+n(n-1)(n-2)(n-3)$

Several well-known special cases of the polynomials $P_{k}^{n}(x)$ are presented in Table 1. Let be $(x)^{(m)}$ the falling factorial defined by $(x)^{(m)}=x(x-$ 1) $\cdots(x-m+1)$. Then:

$$
P_{k}^{n}(x)=\sum_{j=0}^{k-1}(n)^{(k-j)}\left(\begin{array}{l}
k \\
j
\end{array}\right) x^{j} .
$$

TABLE 1 . The special cases $P_{k}^{n}(x)$

\begin{tabular}{|lll|}
\hline$P_{k}^{n}(x)$ & sequences & in $[5]$ \\
\hline$P_{k}^{1}(2)$ & $0,1,4,12,32,80, \ldots$ & $A 001787$ \\
\hline$P_{k}^{1}(3)$ & $0,1,6,27,108,405, \ldots$ & $A 027471$ \\
\hline$P_{k}^{1}(4)$ & $0,1,8,48,256,1280, \ldots$ & $A 002697$ \\
\hline$P_{k}^{2}(1)$ & $0,2,6,12,20,30, \ldots$ & $A 002378$ \\
\hline$P_{k}^{3}(1)$ & $0,3,12,33,72,135, \ldots$ & $A 054602$ \\
\hline$P_{2}^{n}(2)$ & $0,4,10,18,28,40, \ldots$ & $A 028552$ \\
\hline$P_{2}^{n}(3)$ & $0,6,14,24,36,50, \ldots$ & $A 028557$ \\
\hline
\end{tabular}


Note 2. Since

$$
\lim _{i \rightarrow \infty} \frac{(i+1)_{n}}{(i)_{n}}=\lim _{i \rightarrow \infty} \frac{(i+n) !(i-1) !}{(i+n-1) ! i !}=1
$$

the expansion (8) converges for $|x|<1$ and (9) for each $x \in \mathbb{R}$.

It is clear that the formula (9) could be rewritten in the representation of the $e^{x} x^{n}$ function, since there exists the following relationship

$$
\left[e^{x} x^{n}\right]^{(n-1)}=x e^{x}\left[x^{n-1}+P_{n-1}^{n}(x)\right]
$$

between $e^{x} x^{n}$ and the polynomials $P_{k}^{n}(x)$.

\section{REFERENCES}

[1] L. Comtet, Advanced Combinatorics; The art of finite and infinite expansions, D. Reidel Publ. Co., Boston, 1974.

[2] A. Erdélyi, Asymptotic expansions, Dover, New York, 1987.

[3] R. Graham, D. Knuth and I. O. Patashnik, Concrete mathematics, Reading, MA: Addison-Wesley, 1989.

[4] N.E. Nørlund, Hypergeometric function, Acta Math. 94 (1955), 289-349.

[5] N.J.A. Sloane, The On-Linea Encyclopedia of Integer Sequence, published elec. at http://www.research.att.com/ njas/sequences/

[6] H.S. Wilf, Generatingfunctionology, Academic Press, New York, 1990.

Aleksandar Petojević

FACULTY OF EDUCATION

UNIVERSity OF Novi SAD

PODGORIČKA 4

25000 SOMBOR

SERBIA

E-mail address: apetoje@ptt.rs 